\title{
New Physics Working Group Summary of LCWS2000@FNAL
}

\author{
C. S. $\operatorname{Kim}^{1}$ \\ Department of Physics and IPAP, Yonsei University, Seoul 120-749, Korea
}

\begin{abstract}
Here I summarize P5-WG (New/Alternative Physics Working Group) of LCWS2000@FNAL, held on Oct 24-28, 2000. There were 13 talks altogether, 7 talks on collider signals from new particles/interactions and 6 talks on extra dimensional physics. We had very active and hot discussions among participants for those new/alternative physics/ideas. In bottom table, I show those 13 speakers' names and titles.
\end{abstract}

\section{INTRODUCTION}

On the first day of the Workshop, Komamiya asked, in his talk on "Chages to Participants", to our P5-WG (New/Alternative Physics Working Group) to investigate New and Crazy Ideas. How crazy is really crazy? Who are those 13 speakers

1) kim@kimcs.yonsei.ac.kr, cskim@pheno.physics.wisc.edu

TABLE 1. Who are the speakers with new ideas?

\begin{tabular}{ll} 
Collider Speakers & Titles \\
\hline D. Dominici & Signals of new vector resonances [1] \\
P. Kalyniak & Discovery and identification of $W^{\prime}[2]$ \\
S. Riemann & New physics in fermion pair production [3] \\
M. Battaglia & Direct search of $Z^{\prime}$ at CLIC [4] \\
V.A. Ilyin & Potentials of LC in stoponium searches [5] \\
T. Han & Higgs-gauge boson couplings with CP violation [6] \\
C. Heusch & Finding heavy Majorana neutrinos in LC [7] \\
\hline \hline ED Speakers & Titles \\
\hline J. Lykken & Search for Maximal Weirdness [8] \\
J. Heweet & Signals of non-commutative field theories [9] \\
H. Davoudiasl & Probing geometry of the universe at NLC [10] \\
T. Rizzo & Probing RS Warped ED signals at LC [11] \\
T. Takeuchi & Universal torsion induced interactions from ED [12] \\
H.C. Cheng & Electroweak symmetry breaking and ED [13] \\
\hline
\end{tabular}


with new ideas? Please look at next table for the speakers and titles.

Followings are the list of really remarkable ideas:

- Planck scale on your finger tip?

- Natural composite Higgs and EWSB?

- Dynamical symmetry breaking and BESS?

- $W^{\prime}, Z^{\prime}$, heavy Majorana neutrinos?

- Geometry of the universe and universal torsion?

- CP-violationg Higgs-gauge boson couplings?

- non-commutative field theory?

- Maximal weirdness?

As you can see easily, the subjects are changing wildly from each talk, and the summarizing those all talks would be an almost impossible task. In next section, I summarize the talks on signals of new particles/interactions at future colliders. Then, I summarize the talks on physics of Extra Dimension (ED). In last section, I comment on possible violations of CTP and Equivalence Principle.

\section{NEW PARTICLES/INTERACTIONS SIGNALS}

Study of Degenerate-BESS [1]. The degenerate BESS model (D-BESS) [14] is a realization of dynamical electroweak symmetry breaking with decoupling. The D-BESS model introduces two new triplets of gauge bosons, which are almost degenerate in mass, $\left(L^{ \pm}, L_{3}\right),\left(R^{ \pm}, R_{3}\right)$. The extra parameters are a new gauge coupling constant $g^{\prime \prime}$ and a mass parameter $M$, related to the scale of the underlying symmetry breaking sector. In the charged sector the $R^{ \pm}$fields are not mixed and $M_{R^{ \pm}}=M$, while $M_{L^{ \pm}} \simeq M\left(1+x^{2}\right)$ where $x=g / g^{\prime \prime}$ with $g$ the usual $S U(2)_{W}$ gauge coupling constant. The $L_{3}, R_{3}$ masses are given by $M_{L_{3}} \simeq M\left(1+x^{2}\right), \quad M_{R_{3}} \simeq M\left(1+x^{2} \tan ^{2} \theta\right)$ where $\tan \theta=s_{\theta} / c_{\theta}=g^{\prime} / g$ and $g^{\prime}$ is the usual $U(1)_{Y}$ gauge coupling constant. These resonances are narrow and almost degenerate in mass with $\Gamma_{L_{3}} / M \simeq 0.068 x^{2}$ and $\Gamma_{R_{3}} / M \simeq 0.01 x^{2}$, while the neutral mass splitting is: $\Delta M / M=\left(M_{L_{3}}-M_{R_{3}}\right) / M \simeq\left(1-\tan ^{2} \theta\right) x^{2} \simeq 0.70 x^{2}$. This model respects the existing stringent bounds from electroweak precision data since the $S, T, U$ (or $\left.\epsilon_{1}, \epsilon_{2}, \epsilon_{3}\right)$ parameters vanish at the leading order due to an additional custodial symmetry. Therefore, the precision electroweak data only set loose bounds on the parameter space of the model, comparable to those from the direct search at the Tevatron [14]. Future hadron colliders may be able to discover these new resonances which are produced through a $q \bar{q}$ annihilation and which decay in the leptonic channel $q \bar{q}^{\prime} \rightarrow L^{ \pm}, W^{ \pm} \rightarrow\left(e \nu_{e}\right) \mu \nu_{\mu}$ and 
TABLE 2. Sensitivity to $L_{3}$ and $R_{3}$ production at the LHC and CLIC for $L=100(500) \mathrm{fb}^{-1}$ with $M=1,2(3) \mathrm{TeV}$ at LHC and $L=1000 \mathrm{fb}^{-1}$ at CLIC.

\begin{tabular}{ccccccc}
\hline$g / g^{\prime \prime}$ & $\begin{array}{c}M \\
(\mathrm{GeV})\end{array}$ & $\begin{array}{c}\Gamma_{L_{3}} \\
(\mathrm{GeV})\end{array}$ & $\begin{array}{c}\Gamma_{R_{3}} \\
(\mathrm{GeV})\end{array}$ & $\begin{array}{c}S / \sqrt{S+B} \\
\text { LHC }(e+\mu)\end{array}$ & $\begin{array}{c}S / \sqrt{S+B} \\
\text { CLIC (hadrons) }\end{array}$ & $\begin{array}{c}\Delta M \\
\text { CLIC }\end{array}$ \\
\hline 0.1 & 1000 & 0.7 & 0.1 & 17.3 & & \\
0.2 & 1000 & 2.8 & 0.4 & 44.7 & & \\
\hline 0.1 & 2000 & 1.4 & 0.2 & 3.7 & & \\
0.2 & 2000 & 5.6 & 0.8 & 8.8 & 62 & $23.20 \pm .06$ \\
\hline 0.1 & 3000 & 2.0 & 0.3 & $(3.4)$ & 152 & $83.50 \pm .02$ \\
0.2 & 3000 & 8.2 & 1.2 & $(6.6)$ & &
\end{tabular}

$q \bar{q} \rightarrow L_{3}, R_{3}, Z, \gamma \rightarrow\left(e^{+} e^{-}\right) \mu^{+} \mu^{-}$. The relevant observables are the di-lepton transverse and invariant masses. The main backgrounds, left to these channels after the lepton isolation cuts, are the Drell-Yan processes with SM gauge bosons exchange in the electron and muon channel. Results are given in Table 2 for the combined electron and muon channels for $L=100 \mathrm{fb}^{-1}$. Results are given for an integrated luminosity of $500 \mathrm{fb}^{-1}$ assuming $M=3 \mathrm{TeV}$. The discovery limit at LHC with $L=100 \mathrm{fb}^{-1}$ is $M \sim 2 \mathrm{TeV}$ with $g / g^{\prime \prime}=0.1$. Beyond discovery, the possibility to disentangle the double peak structure depends strongly on $g / g^{\prime \prime}$ and smoothly on the mass. A lower energy LC can also probe this multi-TeV region through the virtual effects in the cross-sections for $e^{+} e^{-} \rightarrow L_{3}, R_{3}, Z, \gamma \rightarrow f \bar{f}$. Due to the presence of new spin-one resonances the annihilation channel in $f \bar{f}$ and $W^{+} W^{-}$is more efficient than the fusion channel. In the case of D-BESS, the $L_{3}$ and $R_{3}$ states are not strongly coupled to $W W$ making the $f \bar{f}$ final states the most favorable channel for discovery.

Investigation of $W^{\prime}$ Bosons at $L C$ [2]. Extra gauge bosons are a fundamental part of many extensions of the Standard Model (SM). Extensive investigations of the neutral $Z^{\prime}$ exist in the literature but the charged $W^{\prime}$ has been less well studied. We focus here on the possibility of finding a $W^{\prime}$ and measuring its couplings to fermions at a high energy $e^{+} e^{-}$collider. The models (see references in $[15,16])$ we consider are the Left-Right symmetric model (LRM) based on the gauge group $S U(2)_{L} \times S U(2)_{R} \times U(1)_{B-L}$, the Un-Unified model (UUM) based on the gauge group $S U(2)_{q} \times S U(2)_{l} \times U(1)_{Y}$ where the quarks and leptons each transform under their own $S U(2)$, a Third Family Model (3FM) based on the gauge group $S U(2)_{h} \times S U(2)_{l} \times U(1)_{Y}$ where the quarks and leptons of the third (heavy) family transform under a separate group [17] and the KK model (KK) which contains the Kaluza-Klein excitations of the SM gauge bosons that are a possible consequence of theories with large extra dimensions. We also consider a $W^{\prime}$ with SM couplings as a benchmark which we denote as the Sequential Standard Model (SSM). The particular processes which we study are $e^{+} e^{-} \rightarrow \nu \bar{\nu} \gamma$ and $e \gamma \rightarrow \nu q+X$. The first process we consider is $e^{+} e^{-} \rightarrow \nu \bar{\nu} \gamma$, which includes contributions from both $W^{\prime}$ 's and $Z^{\prime}$ 's. The kinematic variables of interest are the photon's energy, $E_{\gamma}$, and its 
angle relative to the incident electron, $\theta_{\gamma}$, both defined in the $e^{+} e^{-}$centre-of-mass frame. To take into account finite detector acceptance we imposed constraints on the kinematic variables such that $E_{\gamma} \geq 10 \mathrm{GeV}$ and $10^{\circ} \leq \theta_{\gamma} \leq 170^{\circ}$. The most serious background is radiative Bhabha scattering where the scattered $e^{+}$and $e^{-}$ go undetected down the beam pipe. We suppress this background by restricting the photon's transverse momentum to $p_{T}^{\gamma}>\sqrt{s} \sin \theta_{\gamma} \sin \theta_{v} /\left(\sin \theta_{\gamma}+\sin \theta_{v}\right)$ where $\theta_{v}=25 \mathrm{mrad}$ and is the minimum angle to which the veto detectors may observe electrons or positrons. There are also higher order backgrounds which cannot be suppressed, such as $e^{+} e^{-} \rightarrow \nu \bar{\nu} \nu^{\prime} \bar{\nu}^{\prime} \gamma$, which would have to be included in an analysis of data. The second process investigated is $e \gamma \rightarrow \nu q+X$, using photon spectra from both the Weizsacker Williams process and from a backscattered laser. This process contains contributions from only $W^{\prime \prime}$ s and not from $Z^{\prime}$ 's. Starting with the process $e \gamma \rightarrow \nu q \bar{q}$ the $W^{\prime}$ contributions can be enhanced by imposing the kinematic cut that either the $q$ or $\bar{q}$ is collinear to the beam axis. In this kinematic region the process $e \gamma \rightarrow \nu q \bar{q}$ is approximated quite well by the simpler process $e q \rightarrow \nu q^{\prime}$ where the quark is described by the quark parton content of the photon, the so-called resolved photon approximation. We use the process $e q \rightarrow \nu q^{\prime}$ to obtain our limits as it is computationally much faster and the limits obtained in this approximation are in good agreement with those using the full process.

Study of $Z^{\prime}$ at CLIC [4]. The $Z^{\prime}$ mass and width can be determined by performing either an energy scan, like the $Z^{0}$ scan performed at LEP/SLC and also foreseen for the $t \bar{t}$ threshold, or an auto-scan, by tuning the collision energy just above the top of the resonance and profiting of the long tail of the luminosity spectrum to probe the resonance peak. For the first method both di-jet and di-lepton final states can be considered, while for the auto-scan only $\mu^{+} \mu^{-}$final states may provide with the necessary accuracy for the $Z^{\prime}$ energy. $e^{+} e^{-} \rightarrow Z^{\prime}$ events have been generated for $M_{Z^{\prime}}=3 \mathrm{TeV}$, including the effects of ISR, luminosity spectrum and $\gamma \gamma$ backgrounds, assuming SM-like couplings, corresponding to a total width $\Gamma_{Z_{S M}^{\prime}} \simeq 90 \mathrm{GeV}$. A data set of $1000 \mathrm{fb}^{-1}$ has been assumed for the CLIC.01 beam parameters and of $400 \mathrm{fb}^{-1}$ for CLIC.02, corresponding to one year ( = 10 $\mathrm{s}$ ) of operation at nominal luminosity. This has been shared in a 3 to 7 points scan and $M_{Z^{\prime}}, \Gamma\left(Z^{\prime}\right) / \Gamma_{S M}$ and $\sigma_{\text {peak }}$ have been extracted from a $\chi^{2}$ fit to the predicted cross

TABLE 3. Results of the fits for the cross section scan of a $Z_{S M}^{\prime}$ obtained by assuming no radiation and ISR with the effects of two different optimization of the CLIC luminosity spectrum.

\begin{tabular}{lccc}
\hline Observable & Breit Wigner & CLIC.01 & CLIC.02 \\
\hline$M_{Z^{\prime}}(\mathrm{GeV})$ & $3000 \pm .12$ & \pm .15 & \pm .21 \\
$\Gamma\left(Z^{\prime}\right) / \Gamma_{S M}$ & $1 . \pm .001$ & \pm .003 & \pm .004 \\
$\sigma_{\text {peak }}^{\text {eff }}(\mathrm{fb})$ & $1493 \pm 2.0$ & $564 \pm 1.7$ & $669 \pm 2.9$ \\
\hline
\end{tabular}


section behaviour for different mass and width values. The dilution of the analysing power due to the beam energy spread is appreciable, as can be seen by comparing the statistical accuracy from a fit to the pure Born cross section to after including ISR and beamstrahlung effects. Still, the relative statistical accuracies are better than $10^{-4}$ on the mass and $5 \times 10^{-3}$ on the width. Sources of systematics from the knowledge of the shape of the luminosity spectrum have also been estimated. In order to keep $\sigma_{\text {syst }} \leq \sigma_{\text {stat }}$ it is necessary to control $N_{\gamma}$ to better than $5 \%$ and the fraction $\mathcal{F}$ of collisions at $\sqrt{s}<0.995 \sqrt{s_{0}}$ to about $1 \%$.

Stoponium Searches at $L C$ [5]. Search potentials are estimated for stoponium ( $S$ - bound states of the $t$-quark superpartner), considering the $e^{+} e^{-}$and the $\gamma \gamma$ (Photon Linear Collider-PLC) options. The stop bound state can be described as a quasistationary system with binding energy $\sim 1 \mathrm{GeV}$ for $M_{S}=200-600 \mathrm{GeV}$, if the formation process is faster than destroying one. The SUSY scenario, where tree-level stop decays are somehow suppressed and, therefore, stop decay cannot destroy the stoponium formation, is not an exceptional case (see discussions in $[5,18])$. PLC could be the best machine for discovery of these new narrow strong resonances. Thousands of stoponiums can be produced per $100 \mathrm{fb}^{-1} 1$ in the high energy peak. In the case of scenarios when stoponium decays mainly into two gluons the signal/background ratio is about 1/4. In addition the decay channel $S \rightarrow h h$ could be seen with high significance. Thus, several weeks run is sufficient for the discovery of stoponium, if its mass is approximately known (e.g. from observation of direct stops production at LHC). Then, in MSSM scenarios with dominant $S \rightarrow h h$ decay PLC shows excellent possibilities to discover stoponium, practically immediately after beginning of operating. The signal significance of stoponium events is shown in various channels for the MSSM scenarios with $S \rightarrow g g$ decay mode being dominant, and the total number of stoponium events in case when dominant decay channel is $S \rightarrow h h$. The $e^{+} e^{-}$option also has good discovery prospects but only in the case of the second scenario with dominant, with hundreds of events per 100 $\mathrm{fb}^{-1}$. Interesting possibility appears in the case when the resonance is seated on $0.1 \%$ width luminosity peak - one could resolve the stoponium exited states.

Detection of Heavy Majorana Neutrinos [7]. As argued for years, a TeVlevel $e^{-} e^{-}$collider has the unique capability of producing a "quasi-elastic" back-toback emitted $W^{-}$pair after exchanging a $t$-chanel exchanged Majorana neutrino. A careful investigation of the relevant parameter space led us to come up with respectable counting rates if the electrons are both left-handed and exchange a Majorana neutrino with mass of order $m_{W}<\sqrt{s}<m_{N}$, where $N$ stands for the heavy neutrino. The cross section becomes [19]

$$
\sigma\left(\ell_{L}^{-} \ell_{L}^{-} \rightarrow W^{-} W^{-}\right) ; \mathcal{N} \simeq \frac{1}{M(\mathrm{TeV})^{2}}\left(\frac{s}{M^{2}}\right)^{2}\left|\frac{U_{e i}^{2}}{4 \pi}\right|\left(4 \cdot 10^{5} \mathrm{fb}\right) .
$$

The mixing parameters $U_{e} i$, where the index $i$ stands for one of at least two heavy neutrinos, are constrained by low-energy rare lepton decays to the values $\left(U_{e i}\right)^{2} \times$ 
$(2-40) 10^{-4}$. The final-state signatures are spectacular, cannot be missed, and are easily distinguished from background interactions. Furthermore, any emerging signal must vanish as soon as the incoming electron helicity is changed. It has been argued by a number of authors that the non-observation of neutrinoless double beta decay ought to be interpreted as precluding the potential existence of the heavy Majorana neutrinos the Linear Collider will search for. This notion rests on the misconception that our reaction (1) is tantamount to "inverse neutrinoless double beta decay". In fact, this nuclear decay, on the quark-lepton level, can happen only if two d quarks from two different neutrons inside the parent nucleus approach to within $\left(m_{N}\right)^{-}$, or some $10^{-16} \mathrm{~cm}$; it is constrained by the color Coulomb hard-core repulsion. A careful estimate of several scenarios leads to suppression factors of order $\sim 200$, well beyond what would preclude a possible observation of the double beta decay in question. For detection of Majorana neutrinos at $e-\mu$ colliders, please look Ref. [20].

\section{PHYSICS WITH EXTRA DIMENSION}

$R S$ Phenomenolgy at $L C[10,11]$. Randall and Sundrum(RS) [21] have recently proposed a novel approach in dealing with the hierarchy problem wherein an exponential warp factor arises from a 5-d non-factorizable geometry based on a slice of $\mathrm{AdS}_{5}$ space. Here, two 3-branes sit at the orbifold fixed points $y=0$ (Plank brane) and $y=\pi r_{c}$ (SM or TeV brane) with equal and opposite tensions with the $\mathrm{AdS}_{5}$ space between them. The model contains no large parameter hierarchies with $\bar{M}_{P l}$, the 5-d Planck scale, $M_{5}$, and the AdS curvature parameter, $k$, being of qualitatively similar magnitudes. TeV scales can be generated on the brane at $y=\pi r_{c}$ if gravity is localized on the other brane and $k r_{c} \simeq 11-12$; indeed in this case the scale of physical processes on the SM brane is found to be given by $\Lambda_{\pi}=\bar{M}_{P l} e^{-k r_{c} \pi}$ which is of order a TeV. Such a model leads to very interesting and predictive phenomenology that can be explored in detail at colliders [22]. In the simplest scenario the SM fields are constrained to lie on the $\mathrm{TeV}$ brane while gravitons can propagate in the bulk in which case only two parameters are necessary to describe the model: $c=k / \bar{M}_{P l}$, which is expected to be near though somewhat less than unity, and $m_{1}=k x_{1} e^{-k r_{c} \pi}$, which is the mass of the first graviton Kaluza-Klein excitation. The masses of the higher excitations are given by $m_{n}=m_{1} x_{n} / x_{1}$, where the $x_{n}$ are roots of the Bessel function $J_{1}\left(x_{n}\right)=0$, and are thus not equally spaced. While the massless zero mode graviton couples in the usual manner as $\left(\bar{M}_{P l}\right)^{-1}$, the tower states instead couple as $\Lambda_{\pi}^{-1}$. The most distinctive prediction of this scenario is the direct production of weak scale graviton resonances at colliders as is shown in Fig. 1 for the case of a linear collider. Note that for fixed mass the width of each resonance is proportional to $c^{2}$; for resonances beyond the first KK excitation, the width grows as $m^{3}$. This explains why resonances with large KK number tend to get smeared out into a continuum. Present searches for graviton resonances at the Tevatron as well as analyses of their indirect contributions to electroweak observ- 

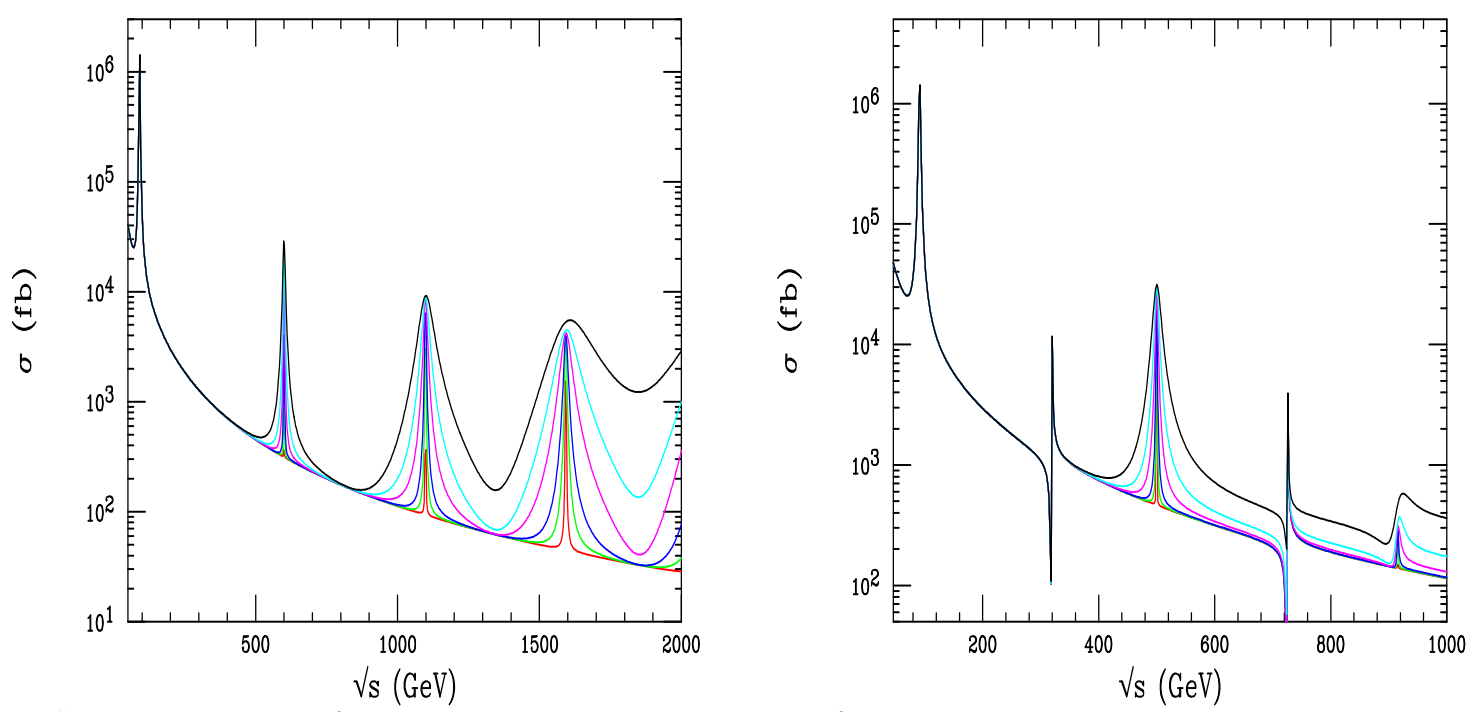

FIGURE 1. The left panel shows the production of KK graviton resonances in the process $e^{+} e^{-} \rightarrow \mu^{+} \mu^{-}$assuming $m_{1}=600 \mathrm{GeV}$ for various values of $c$. In the right panel one sees the simultaneous production of graviton and gauge KK states typical of regions II and III via the process $e^{+} e^{-} \rightarrow \mu^{+} \mu^{-}$.

ables already place significant constraints on the $c-m_{1}$ plane. When combined with our theoretical prejudices the complete allowed region for the RS model is shown in Fig. 1 in comparison to the reach of the LHC. Even given some fuzziness in our prejudices it is apparent that the LHC should cover the entire RS parameter space either by discovering a graviton resonance or excluding the model. If the SM gauge fields alone are allowed to propagate in the bulk then it can be shown that the gauge KK excitations couple much more strongly to the remaining wall fields than do the zero modes [22] by a factor $\simeq \sqrt{2 \pi k r_{c}}$. The exchange and mixing of these modes contribute to the electroweak observables and result in a bound $\Lambda_{\pi}>100 \mathrm{TeV}$ which is perhaps too high to claim a solution to the hierarchy problem. This strong bound can be alleviated by also placing the SM fermions in the bulk as well with the Higgs field remaining on the wall for a number of technical reasons [22]. For simplicity and to avoid FCNC we assume that all SM fermions have an identical 5 -d mass $m_{5 d}=k \nu$, with $\nu$ a parameter of order unity. Specifying $\nu$ and $m_{1}$ for the graviton determines all of the KK masses with fermion excitations always more massive than gauge excitations and are approximately linear functions of $|\nu+1 / 2|$. For the phenomenology of Large Extra Dimensional (LED) physics [23], please look at [24] for single graviton emission processes as missing energy events and the indirect effects of the massive graviton exchange on various collider experiments.

Torsion Induced Interaction in LED [12]. The general situation, in which the Lorentz group is gauged as well, will give rise to an antisymmetric part to the connection coefficient, the torsion tensor. This general situation obtains in the 
presence of intrinsic spin; the torsion tensor is then coupled to the intrinsic spin current, which then represents yet another source of gravity. Since this current cannot be eliminated by a choice of coordinates, the situation breaks the strong form of the equivalence principle. It is possible to require that the torsion tensor still vanishes, but this demand will need to be preserved under radiative corrections by invoking additional symmetries. Here the consequences of torsion in the context of a model with large extra dimensions are considered. Allowing the torsion tensor $T_{\beta \gamma}^{\alpha}=\tilde{\Gamma}_{\beta \gamma}^{\alpha}-\tilde{\Gamma}_{\gamma \beta}^{\alpha}$ to be non-zero introduces an extra piece into the gravitational connection

$$
\tilde{\Gamma}_{\beta \gamma}^{\alpha}=\Gamma_{\beta \gamma}^{\alpha}+K_{\beta \gamma}^{\alpha},
$$

where $\Gamma_{\beta \gamma}^{\alpha}$ is the usual metric contribution, and $K_{\alpha \beta \gamma}=\frac{1}{2}\left(T_{\alpha \beta \gamma}-T_{\beta \alpha \gamma}-T_{\gamma \alpha \beta}\right)$ is known as the contorsion tensor. The action of the model is given by

$$
\begin{aligned}
& S=-\frac{1}{\hat{\kappa}^{2}} \int d^{4+n} x \sqrt{\left|\hat{g}_{4+n}\right|} \tilde{R} \\
& +\int d^{4} x \sqrt{\left|\hat{g}_{4}\right|} \frac{i}{2}\left[\bar{\Psi} \gamma^{\mu} \tilde{\nabla}_{\mu} \Psi-\left(\tilde{\nabla}_{\mu} \bar{\Psi}\right) \gamma^{\mu} \Psi+2 i M \bar{\Psi} \Psi\right] .
\end{aligned}
$$

Here $\hat{\kappa}^{2}=16 \pi G_{N}^{(4+n)}, \tilde{R}$ is the $4+n$ dimensional scalar curvature, and $\hat{g}_{4+n}$ and $\hat{g}_{4}$ are respectively the $4+n$ and 4 -dimensional (induced) metric determinants. Elimination of torsion from the action by imposing the equations of motion results in $[25]$ :

$$
\begin{aligned}
S= & -\frac{1}{\hat{\kappa}^{2}} \int d^{4+n} x \sqrt{\left|\hat{g}_{4+n}\right|} R \\
& +\int d^{4} x \sqrt{\left|\hat{g}_{4}\right|}\left[\bar{\Psi}\left(i \gamma^{\mu} \nabla_{\mu}-M\right) \Psi+\frac{3}{32} \frac{\sqrt{\left|\hat{g}_{4}\right|}}{\sqrt{\left|\hat{g}_{4+n}\right|}} \hat{\kappa}^{2}\left(\bar{\Psi} \gamma_{\mu} \gamma_{5} \Psi\right)^{2} \delta^{(n)}(0)\right] .
\end{aligned}
$$

where $R$ is the torsion-free curvature. The delta-function appearing in this expression should be regularized to account for a finite wall thickness. As a result, the leading $\mathcal{O}\left(\hat{\kappa}^{2}\right)$ torsion contribution to the action is given by

$$
\Delta S=\int d^{4} x \frac{3 \pi}{n M_{S}^{2}}\left[\sum_{j} \bar{\Psi}_{j} \gamma_{\mu} \gamma_{5} \Psi_{j}\right]^{2},
$$

where $j$ runs over all fermions existing on the wall. The expansion in $\hat{\kappa}$ is expected to be valid provided the typical energy $E$ of a physical process is below the cutoff scale $M_{S}$. The torsion induced contact interaction Eq. (5) can be constrained through its effect on $Z$-pole electroweak observables. The corrections shifts the $Z$-couplings. Performing a global fit to the LEP/SLD electroweak observables will lead to a constraint on $Z$-couplings, which in turn will give us a limit on $M_{S}$. 
Extroweak Symmetry Breaking and LED [13]. The electroweak symmetry may be broken by a composite Higgs which arise naturally as a bound state of the top quark if the standard model gauge fields and fermions propagate in extra dimenions. The top quark mass and the Higgs mass can be predicted from the infrared fixed points of the renormalization group equations. The top quark mass is in good agreement with the experimental value, and the Higgs boson mass is predicted to be $\sim 200 \mathrm{GeV}[26]$. The bounds on the compactification scale can be quite low if all standard model fields propagate in the same extra dimensions due to the momentum conservation in extra dimensions. The current lower limits are about $300 \mathrm{GeV}$ for one extra dimensions and $400-800 \mathrm{GeV}$ for two extra dimensions. The future collider experiments may either discover the Kaluza-Klein (KK) states of the standard model fields or raise their mass limits significantly. There may also be some other light bound states which could be observed at upcoming collider experiments. Compared with the usual four-dimensional dynamical electroweak symmetry breaking (EWSB) models, the higher-dimensional model has the advantage that the binding force can be the SM gauge interactions themselves, without the need of introducing new strong interactions. In addition, it also gives a prediction of the top quark mass naturally in the right range. In the minimal four-dimensional top quark condensate model, the top quark is too heavy, $\sim 600$ $\mathrm{GeV}$, if the compositeness scale is in the $\mathrm{TeV}$ range. With extra dimensions, the KK excitations of the top quark also participate in the EWSB, so the top quark mass can be smaller. Another way of understanding of the top Yukawa coupling being $\sim 1$ instead of the strong coupling value $\sim 4 \pi$ is that (the zero mode of) the top quark coupling receives a volume dilution factor because it propagates in extra dimensions. In fact, the top quark mass can be predicted quite insensitively to the cutoff because of the infrared fixed point behavior of the renormalization group (RG) evaluation. The infrared fixed point is rapidly approached due to the power-law running in extra-dimensional theories. even though the cutoff scale is not much higher the the weak scale. Similarly, the Higgs self-coupling also receives the extra-dimensional volume suppression. As a result, the physical Higgs boson is relatively light, $\sim 200 \mathrm{GeV}$, in contrast with the usual strongly coupled four-dimensional models. It is also governed by the infrared fixed point of the RG equations.

\section{COMMENTS ON GRAVITY}

As summarized in previous sections, the main theme of our Working Group was "How precisely has Gravity been probed microscopically and macroscopically?" Here I note about it from two different approaches.

CPT violation from a tilted brane [27]. The tilted brane in large extra dimension can be described by a low energy effective theory. In this theory, the graviphoton $\mathcal{A}_{\mu}(x)$ obtains a mass $m_{\mathcal{A}} \sim 1 \mathrm{~mm}^{-1}$ by eating up the corresponding 
Goldstone mode. After integrating out the graviphoton, the effective theory for the other pseudo-Nambu-Goldstone boson(s) $\chi$ describing the dynamics of the brane is represented by the four-dimensional Lagrangian density

$$
\mathcal{L}_{\text {brane }}=g^{\mu \nu} \partial_{\mu} \chi \partial_{\nu} \chi
$$

where $g_{\mu \nu}$ is the induced metric on the brane. The tilted brane solution to the equation of motion $\partial^{2} \chi=0$ is

$$
\chi=\sqrt{T} \alpha x^{j}
$$

where $\hat{x}^{j}$ is the direction along which the tilting occurs, $T$ is the brane tension, i.e., the energy per 3 -space unit volume, and $\alpha$ is the angle of the tilting. The tiny tilting angle $\alpha$ causes the brane to be "stretched" by the factor of $1+\alpha^{2} / 2$. On the other hand, $\alpha=T^{-1 / 2} \partial \chi / \partial x^{j}$ by (7). This implies that the induced metric $g_{\mu \nu}$ and the flat (untilted) metric $g_{\mu \nu}^{(0)}$ are related

$$
g_{\mu \nu}=g_{\mu \nu}^{(0)}+\frac{1}{2} T^{-1} \partial_{\mu} \chi \partial_{\nu} \chi
$$

An analogous relation for the basis 4 -vectors $e_{a}$ on the tilted brane

$$
e_{a}^{\mu}=g_{a}^{(0) \mu}+\frac{1}{4} T^{-1} \partial^{\mu} \chi \partial_{a} \chi
$$

follows from (8) due to $e_{a} \cdot e_{b}=g_{a b}$. The kinetic term for the fermionic fields on the tilted brane involves $\not_{\text {tilted }}=e_{a}^{\mu} \gamma^{a} \partial_{\mu}$. Therefore, when using (9), the kinetic energy term in the tilted brane background can be rewritten as

$$
\mathcal{L}_{\text {kin. }}=(\bar{\psi} \not \partial \psi)_{\text {tilted }}=\bar{\psi} \gamma^{\mu} \partial_{\mu} \psi+\frac{1}{4} T^{-1}\left(\partial^{\mu} \chi \partial_{\nu} \chi\right)\left(\bar{\psi} \gamma^{\nu} \partial_{\mu} \psi\right)
$$

where all the derivatives are in the flat metric. If we now expand around the tilted brane solution (7), i.e., $\chi=\sqrt{T} \alpha x^{j}+\delta \chi$, we obtain from the last term of (10) interaction terms which break the Lorentz and rotational invariance

$$
\delta \mathcal{L}=\frac{1}{4} T^{-1 / 2} \alpha \partial_{\nu}(\delta \chi)\left[\bar{\psi}\left(\gamma^{\nu} \partial^{j}+\gamma^{j} \partial^{\nu}\right) \psi\right]+\left.\frac{1}{4} \alpha^{2} \bar{\psi} \gamma^{\nu} \partial^{\mu} \psi\right|_{\nu=\mu=j}
$$

The first term $(\propto \alpha)$, in addition, violates CPT, because $\partial_{\nu}(\delta \chi)$ is odd and the term in $[\ldots]$ is even under CPT.

Neutrino oscillation from Violation of Equivalence Principle [28]. If $\gamma_{\nu} \equiv \sqrt{1-\beta_{\nu}^{2}}$ is flavor dependent, then different neutrinos will undergo different gravitational time delays when passing through the same gravitational potential and thereby acquire different phase shifts. These phase shifts are observable owing to the difference in the particle bases that diagonalize the weak and the gravitational 
interactions. As a consequence, a $\nu_{e}$ will be able to oscillate into $\nu_{\mu}$. In the absence of non-gravitational interactions, the properties of a spin-1/2 particle in a specified gravitational field, $G_{\alpha \beta}$, are usually described to first order (linearized therory) by the interaction Lagrangian density

$$
\mathcal{L}_{\text {int }}=i \frac{f}{4} G^{\alpha \beta}\left[\bar{\psi} \gamma_{\alpha} \partial_{\beta} \psi-\left(\partial_{\alpha} \bar{\psi}\right) \gamma_{\beta} \psi\right]
$$

where $f=\sqrt{8 \pi G_{N}}, G_{N}$ is Newton's constant and the metric of flat space is $g_{\alpha \beta}=(+1,-1,-1,-1)$. An interaction of the above form but one which allows the neutrinos $\nu_{1}$ and $\nu_{2}$ to couple to gravity with different strengths $f_{1}$ and $f_{2}$ can be postulated. Then, the postulated interaction leads to the equations of motion for the massless neutrino fields, $\nu_{j}$,

$$
\left[\left(g^{\alpha \beta}+\frac{f_{j}}{2} G^{\alpha \beta}\right) \gamma_{\alpha} \partial_{\beta}+\frac{f_{j}}{4}\left(\partial_{\alpha} G^{\alpha \beta}\right) \gamma_{\beta}\right] \nu_{j}=0, \quad j=1,2, \ldots
$$

In this case, the $\nu_{j}$ satisfy a Klein-Gordon equation,

$$
\left(g^{\alpha \beta}+f_{j} G^{\alpha \beta}\right) \partial_{\alpha} \partial_{\beta} \nu_{j}=0 .
$$

If we assume the gravitational field is determined by a static macroscopic matter distribution in the harmonic gauge, such a field is given in terms of the Newtonian potential $\phi$ by

$$
G_{\alpha \beta}=2 \phi \delta_{\alpha \beta} / f
$$

where $\phi(\infty) \rightarrow 0$. To illustrate the essential properties of the resulting phase shifts, we consider the case of constant $\phi$, where we have the energy-momentum relation

$$
E^{2}\left(1+2 \gamma_{j} \phi\right)=p^{2}\left(1-2 \gamma_{j} \phi\right) .
$$

For the simple case of two neutrinos, this implies that, after traversing a distance $l$, the two components, $\left(\nu_{1}, \nu_{2}\right)$, of a state $\nu_{e}$ will develop a phase difference of $\delta=2\left(\gamma_{1}-\gamma_{2}\right) \phi l p$. If we compare this phase shift with that obtained in the well known case of vacuum oscillations induced by a neutrino mass difference, we find that they are related by the formal connection,

$$
\frac{\Delta m^{2}}{2 E} \rightarrow 2 E|\phi| \Delta \gamma
$$

where $\Delta \gamma \equiv \gamma_{2}-\gamma_{1}$. By analogy, the $\nu_{e}$ survival probability after traversing a distance $l$ is given by

$$
P\left(\nu_{e} \rightarrow \nu_{e}\right)=1-\sin ^{2} 2 \theta_{G} \sin ^{2} \frac{\pi l}{\lambda}, \quad \text { with } \lambda=6.2 \mathrm{~km}\left(\frac{10^{-20}}{|\phi \Delta \gamma|}\right)\left(\frac{10 \mathrm{GeV}}{E}\right) .
$$


Acknowledgements. I would like to thank the co-convenors of P5-WG, S. Tkaczyk and G. Wilson. The work was supported in part by Seo-Am foundation, in part by BK21 Program, SRC Program and Grant No. 2000-1-11100-003-1 of the KOSEF, and in part by the KRF Grants, Project No. 2000-015-DP0077.

\section{REFERENCES}

1. D. Dominici, Proc. of LCWS2000@FNAL, Oct. 2000.

2. P. Kalyniak, Proc. of LCWS2000@FNAL, Oct. 2000.

3. S. Riemann, Proc. of LCWS2000@FNAL, Oct. 2000.

4. M. Battaglia, Proc. of LCWS2000@FNAL, Oct. 2000.

5. V.A. Ilyin, Proc. of LCWS2000@FNAL, Oct. 2000.

6. T. Han, Proc. of LCWS2000@FNAL, Oct. 2000.

7. C. Heusch, Proc. of LCWS2000@FNAL, Oct. 2000.

8. J. Lykken, Proc. of LCWS2000@FNAL, Oct. 2000.

9. J. Heweet, Proc. of LCWS2000@FNAL, Oct. 2000.

10. H. Davoudiasl, Proc. of LCWS2000@FNAL, Oct. 2000.

11. T. Rizzo, Proc. of LCWS2000@FNAL, Oct. 2000.

12. T. Takeuchi, Proc. of LCWS2000@FNAL, Oct. 2000.

13. H.C. Cheng, Proc. of LCWS2000@FNAL, Oct. 2000.

14. R. Casalbuoni et al., Phys. Lett. 349, 533 (1995.)

15. S. Godfrey, P. Kalyniak, B. Kamal, and A. Leike, Phys. Rev. 61, 113009 (2000).

16. S. Godfrey, P. Kalyniak, B. Kamal, M. Doncheski and A. Leike, hep-ph/0008157.

17. S. Chivukula, E. Simmons and J. Terning, Phys. Lett. 331, 383 (1994).

18. M. Drees and M.M. Nojiri, Phys. Rev. 49,, 4595 (1994).

19. C.A. Heusch, P. Minkowski, Phys. Lett. 374, 116 (1996).

20. G. Cvetic, C.S. Kim and C.W. Kim, Phys. Rev. Lett. 82, 4761 (1999); G. Cvetic and C.S. Kim, Phys. Lett. 461, 248 (1999).

21. L. Randall and R. Sundrum, Phys. Rev. Lett. 83, 3370 (1999).

22. H. Davoudiasl, J.L. Hewett and T.G. Rizzo, Phys. Rev. Lett. 84, 2080 (2000); Phys. Lett. B473, 43 (2000).

23. N. Arkani-Hamed, S. Dimopoulos and G. Dvali, Phys. Lett. 429, 263 (1998).

24. E.A. Mirabelli, M. Perelstein and M.E. Peskin, Phys. Rev. Lett. 82, 2236 (1999); J. L. Hewett, Phys. Rev. Lett. 82, 4765 (1999); C. Balazs, H.-J. He, W.W. Repko and C.-P. Yuan, Phys. Rev. Lett. 83, 2112 (1999); K. Cheung and W.-Y. Keung, Phys. Rev. 60, 112003 (1999); K. Agashe and N. G. Deshpande, Phys. Lett. B456, 60 (1999); T. G. Rizzo and J. D. Wells, Phys. Rev. 61, 016007 (2000); K. Y. Lee, H. S. Song and J. Song, Phys. Lett. 464, 82 (1999); C.S. Kim, K.Y. Lee and J.H. Song, hep-ph/0009231.

25. L.N. Chang, O. Lebedev, W. Loinaz and T. Takeuchi, Phys. Rev. Lett. 85, 3765 (2000).

26. H.-C. Cheng, B. A. Dobrescu and C. T. Hill, Nucl. Phys. 589, 249 (2000).

27. G. Cvetic, S.K. Kang, C.S. Kim and K. Lee, Phys. Rev. 62, 057901 (2000).

28. A. Halprin, C.N. Leung and J. Pantaleone, Phys. Rev. 53, 5365 (1996). 